\title{
Molecular mechanisms of breast cancer metastasis by gene expression profile analysis
}

\author{
TIANYING ZHENG, AIJUN WANG, DONGYAN HU and YONGGANG WANG
}

\begin{abstract}
Department of Chemotherapy, Cancer Center, Qilu Hospital of Shandong University, Jinan, Shandong 250012, P.R. China
\end{abstract}
Received September 7, 2016; Accepted May 18, 2017

DOI: $10.3892 / \mathrm{mmr} .2017 .7157$

\begin{abstract}
Metastasis is the main cause of breast cancer-related mortalities. The present study aimed to uncover the relevant molecular mechanisms of breast cancer metastasis and to explore potential biomarkers that may be used for prognosis. Expression profile microarray data GSE8977, which contained 22 stroma samples (15 were from normal breast and 7 were from invasive ductal carcinoma tumor samples), were obtained from the Gene Expression Omnibus database. Following data preprocessing, differentially expressed genes (DEGs) were selected based on analyses conducted using the linear models for microarray analysis package from $\mathrm{R}$ and Bioconductor software. The resulting data were used in subsequent function and pathway enrichment analyses, as well as protein-protein interaction (PPI) network and subnetwork analyses. Transcription factors (TFs) and tumor-associated genes were also identified among the DEGs. A total of 234 DEGs were identified, which were enriched in immune response, cell differentiation and cell adhesion-related functions and pathways. Downregulated DEGs included TFs, such as the proto-oncogene $S P I 1$, pre-B-cell leukemia homeobox 3 $(P B X 3)$ and lymphoid enhancer-binding factor 1 (LEF1), as well as tumor suppressors (TSs), such as capping actin protein, gelsolin like $(C A P G)$ and tumor protein $\mathrm{p} 53$-inducible nuclear protein 1 (TP53INP1). Upregulated DEGs also included TFs and tumor suppressors, consisting of transcription factor 7-like 2 (TCF7L2) and pleiomorphic adenoma gene-like 1 (PLAGL1). DEGs that were identified at the hub nodes in the PPI network and the subnetwork were epidermal growth factor receptor $(E G F R)$ and spleen-associated tyrosine kinase $(S Y K)$, respectively. Several genes crucial in the metastasis of breast cancer were identified, which may serve as potential biomarkers, many of which were associated with cell adhesion,
\end{abstract}

Correspondence to: Dr Yonggang Wang, Department of Chemotherapy, Cancer Center, Qilu Hospital of Shandong University, 107 West Wenhua Road, Jinan, Shandong 250012, P.R. China E-mail: yonggang0809@163.com

Key words: breast cancer metastasis, protein-protein interaction, immune response, cell differentiation, cell adhesion proliferation or immune response, and may influence breast cancer metastasis by regulating these function or pathways.

\section{Introduction}

Breast cancer is a malignant disease that affects women worldwide. In the United States, breast cancer was estimated to account for $29 \%$ of all new cancer cases and $15 \%$ of all cancer-related mortality among women in 2014 (1). The major cause of these mortalities was not the primary breast cancer tumor, but the metastases at distant sites; in most patients with breast cancer, the tumor was able to metastasize to different organs, such as bone, lungs and brain (2-4). In a number of patients, breast cancer has been demonstrated to be aggressive and metastasized to a distant site a short time following the detection of primary tumor, whereas in other cases the hallmark of metastases may not be evident for a longer period (5). This feature makes it difficult to identify the risk factors of breast cancer metastasis and to evaluate relevant curative therapies (5). Although mammographic screening may lower the metastasis-related mortality, the method is inappropriate for detection at early stages (6). Therefore, it is urgent to develop effective molecular techniques for the diagnosis and treatment of breast cancer metastasis.

The initial event of metastasis may be the transformation of normal breast stromal cells to cells with oncogenic mutations. The subsequent generation of cancer stromal cells may lead to poor prognosis, as a self-renewing population of stromal cells accumulates the mutations required for tumorigenesis (7). In the transforming growth factor $\beta$ receptor 2 knockout mouse model, stromal fibroblasts were reported to be a major source of elevated TGF $\beta$ expression, which was used to predict early breast cancer metastasis (8). Therefore, breast cancer stromal cells may be considered as attractive therapeutic targets. At present, several molecular targeting therapies have been developed; for example, agents targeting HER2 (such as trastuzumab) have been used to treat patients with metastatic breast cancer in which HER2 is overexpressed (9). P38 mitogenactivated protein kinase has been reported to inhibit breast cancer metastasis through the regulation of stromal expansion (10). In addition, breast cancer metastasis-suppressor 1 was demonstrated to suppress metastasis in multiple organs, such as breast and ovary, by blocking several of the steps involved in the metastatic process (11); however, it was apparently insufficient to use only one gene as a targeted therapy due 
to the complex pathogenesis of cancer metastasis. Therefore, an improved understanding of the molecular mechanisms of metastasis is required to discover as yet unknown target genes.

The present study analyzed expression profile microarray data GSE8977 from the Gene Expression Omnibus (GEO) database, which contained stroma samples from normal and invasive ductal carcinoma (IDC) breast tissue samples, to perform bioinformatics analyses, including differential analysis, enrichment analysis and protein-protein interaction (PPI) network analysis. The present study aimed to identify potentially important gene markers and to gain better insight into the molecular mechanism of breast cancer metastasis.

\section{Materials and methods}

Microarray data. Expression profile GSE8977 was obtained from National Center of Biotechnology Information GEO database (http://www.ncbi.nlm.nih.gov/geo/query/acc .cgi?acc=GSE8977) (12). The profile comprised 22 stroma samples, of which 15 were from normal breast samples (Control group) and 7 were from IDC tumor samples (Tumor group), and was based on the GPL570 platform data (Affymetrix Human Genome U133 Plus 2.0 Array).

Data preprocessing. Raw data underwent preprocessing, such as background correction, quantile normalization and probe summarization, using the robust multi-array average algorithm (13) and the 'affy' package version 1.54 .0 (http://www .bioconductor.org/packages/release/bioc/html/affy.html) in Bioconductor, as previously described (14), and a gene expression matrix was obtained.

Differently expressed gene (DEG) selection. Empirical Bayes test was used to identify DEGs between the two groups, based on the Linear Models for Microarray Analysis ('limma') package version 3.32 .2 of $\mathrm{R}$ (http://www.bioconductor. org/packages/release/bioc/html/limma.html) (15). The selection criteria for significant DEGs were $\mid \log 2$ fold-changel $\geq 1$ and a false discovery rate (FDR)-adjusted $\mathrm{P}$-value $<0.05$.

Enrichment analysis of DEGs. Function enrichment analysis of the identified DEGs was performed with the Gene Ontology (GO) database (http://www.geneontology.org) (16), which included functions on biological process, molecular function and cellular component. The Kyoto Encyclopedia of Genes and Genomes (KEGG) database (http://www.genome .jp/kegg/pathway.html) was used to identify significantly enriched pathways (17). The threshold for significant GO terms and KEGG pathways was $\mathrm{P}<0.01$, based on hypergeometric distribution.

Functional annotation of the corresponding DEGs. The identified DEGs were combined with information from the TRANScription FACtor (TRANSFAC) database version 7.0 (http://www.gene-regulation.com/pub/databases.html) to analyze their potential transcriptional functions and to reveal which were potential transcription factors (TFs). Oncogenes and tumor suppressor (TS) genes were also screened using the TS database (http://bioinfo.mc.vanderbilt.edu/TSGene/) version 2.0 (18) and the Tumor Associated Genes (TAGs) database (http://www.binfo.ncku.edu.tw/TAG/) (19).

Construction of a PPI network and subnetwork. To explore potential interactions of the DEGs on the protein level and to establish a PPI network, DEGs were mapped with the Search Tool for the Retrieval of Interacting Genes (STRING) database version 10.5 (http://string-db.org) (20), with the criterion of a combined score $\geq 0.9$. Only the PPIs that were validated by previous experiments or co-expression analysis, or recorded in relevant databases were screened out to construct the network. In addition, it was required that at least one gene in each PPI should be an identified DEG. Finally, the PPI network was visualized using Cytoscape version 3.5.1 (http://cytoscape.org) software (21).

The BioNet tool (http://www.bioconductor.org/packages/release/bioc/html/BioNet.html) version 1.36.0 in $\mathrm{R}$ was used to extract the subnetwork, based on significant P-values of DEGs (22). FDR-adjusted $\mathrm{P}<0.0001$ was chosen as the cut-off value for subnetwork extraction. KEGG pathway enrichment analysis was performed to reveal potential pathways for genes in the subnetwork, using the aforementioned criterion.

\section{Results}

Analysis of DEGs. Based on the selection criteria, a total of 910 transcripts were identified to be differentially expressed between the two groups, 347 of which were upregulated and 563 were downregulated. These transcripts corresponded to 84 upregulated genes and 150 downregulated genes, giving a total of 234 identified DEGs.

KEGG pathway and GO functional enrichment analyses. KEGG pathway enrichment analysis indicated that the downregulated DEGs were enriched in immune response-related pathways, including Staphylococcus aureus infection, allograft rejection and graft-versus-host disease pathways, as well as pathways related to cell differentiation and cell adhesion, such as hematopoietic cell lineage, osteoclast differentiation and cell adhesion molecules. The upregulated DEGs were enriched in cancer-related pathways, including colorectal, endometrial and thyroid cancer pathways, as well as the adherens junction pathway (Table I).

To gain further insight into the function of the identified DEGs, GO analysis was performed (Table II). The downregulated DEGs were revealed to be highly related to immunoreactions, such as activation of immune response, leukocyte migration and positive regulation of leukocyte activation; additional functions included cell adhesion and protein secretion. The upregulated DEGs were mainly enriched in cell proliferation- and tissue development-related functions, such as muscle cell proliferation, regulation of epithelial cell proliferation and tissue development; as well as cell adhesion-related processes such as regulation of focal adhesion assembly.

Functional annotation of TFs and TAGs. The identified DEGs were analyzed for potential functions as TFs and TAGs (Table III). The analysis indicated that, of the downregulated DEGs, 3 were identified as TFs and 12 as TAGs; of the upregulated DEGs, 6 were indicated to be TFs and 17 were TAGs. 
Table I. Enriched KEGG pathways of the identified DEGs.

\begin{tabular}{|c|c|c|c|c|}
\hline DEG expression & KEGG pathway & $\mathrm{n}$ & Example of identified genes & P-value \\
\hline \multirow[t]{7}{*}{ Downregulated } & Staphylococcus aureus infection & 16 & $\begin{array}{l}\text { ITGB2, FPR1, FPR3, } \\
\text { HLA-DRB3, ITGAM }\end{array}$ & $1.13 \times 10^{-15}$ \\
\hline & Phagosome & 17 & $\begin{array}{l}\text { ITGB2, CORO1A, DRB1, } \\
\text { HLA-DMB, COMP }\end{array}$ & $6.96 \times 10^{-13}$ \\
\hline & Allograft rejection & 10 & $\begin{array}{l}C D 80, C D 86, H L A-D M B, \\
H L A-D Q A 1, H L A-D Q A 2\end{array}$ & $5.97 \times 10^{-12}$ \\
\hline & Cell adhesion molecules & 15 & $\begin{array}{l}C D 4, C D 80, I T G B 2 \\
C D 86, H L A-D M B\end{array}$ & $1.54 \times 10^{-11}$ \\
\hline & Graft-versus-host disease & 10 & $\begin{array}{l}C D 80, C D 86, H L A-D M B, \\
H L A-D Q A 1, H L A-D Q A 2\end{array}$ & $1.85 \times 10^{-11}$ \\
\hline & Osteoclast differentiation & 10 & $\begin{array}{l}L C P 2, L I L R B 1, T Y R O B P, \\
S P I 1, B L N K\end{array}$ & $1.70 \times 10^{-06}$ \\
\hline & Hematopoietic cell lineage & 5 & $\begin{array}{l}C D 4, H L A-D R A, H L A-D R B 1, \\
H L A-D R B 3, I T G A M\end{array}$ & $3.35 \times 10^{-03}$ \\
\hline \multirow[t]{5}{*}{ Upregulated } & Endometrial cancer & 3 & EGFR, TCF7L1, TCF7L2 & $2.25 \times 10^{-03}$ \\
\hline & Colorectal cancer & 3 & MSH6, TCF7L1, TCF7L2 & $3.72 \times 10^{-03}$ \\
\hline & Complement and coagulation cascades & 3 & CFD, PROS1, TFPI & $5.03 \times 10^{-03}$ \\
\hline & Adherens junction & 3 & EGFR, TCF7L1, TCF7L2 & $5.89 \times 10^{-03}$ \\
\hline & Thyroid cancer & 2 & $T C F 7 L 1, T C F 7 L 2$ & $9.41 \times 10^{-03}$ \\
\hline
\end{tabular}

DEG, differentially expressed gene; KEGG, Kyoto Encyclopedia of Genes and Genomes.

Table II. GO term functional enrichment of DEGs.

\begin{tabular}{|c|c|c|c|c|}
\hline DEG expression & GO ID & GO term & $\mathrm{n}$ & P-value \\
\hline \multirow[t]{10}{*}{ Downregulated } & GO:0002253 & Activation of immune response & 38 & $1.26 \times 10^{-25}$ \\
\hline & GO:0050900 & Leukocyte migration & 32 & $3.43 \times 10^{-21}$ \\
\hline & GO:0071345 & Cellular response to cytokine stimulus & 40 & $2.31 \times 10^{-18}$ \\
\hline & GO:0002696 & Positive regulation of leukocyte activation & 29 & $3.33 \times 10^{-16}$ \\
\hline & GO:0006954 & Inflammatory response & 41 & $1.78 \times 10^{-15}$ \\
\hline & GO:0060326 & Cell chemotaxis & 20 & $1.41 \times 10^{-11}$ \\
\hline & GO:0009306 & Protein secretion & 21 & $4.17 \times 10^{-11}$ \\
\hline & GO:0009615 & Response to virus & 25 & $4.29 \times 10^{-11}$ \\
\hline & GO:0007155 & Cell adhesion & 48 & $2.83 \times 10^{-10}$ \\
\hline & GO:0071222 & Cellular response to lipopolysaccharide & 15 & $7.00 \times 10^{-10}$ \\
\hline \multirow[t]{10}{*}{ Upregulated } & GO:0035265 & Organ growth & 6 & $9.06 \times 10^{-06}$ \\
\hline & GO:0031644 & Regulation of neurological system process & 8 & $1.85 \times 10^{-05}$ \\
\hline & GO:0033002 & Muscle cell proliferation & 6 & $2.01 \times 10^{-05}$ \\
\hline & GO:0006790 & Sulfur compound metabolic process & 8 & $3.65 \times 10^{-05}$ \\
\hline & GO:0050678 & Regulation of epithelial cell proliferation & 7 & $1.17 \times 10^{-04}$ \\
\hline & GO:0009888 & Tissue development & 19 & $1.49 \times 10^{-04}$ \\
\hline & GO:0007420 & Brain development & 10 & $4.07 \times 10^{-04}$ \\
\hline & GO:0051893 & Regulation of focal adhesion assembly & 3 & $4.45 \times 10^{-04}$ \\
\hline & GO:0032355 & Response to estradiol stimulus & 4 & $5.27 \times 10^{-04}$ \\
\hline & GO:0019530 & Taurine metabolic process & 2 & $5.05 \times 10^{-01}$ \\
\hline
\end{tabular}


Table III. Functional annotation of the identified DEGs.

\begin{tabular}{|c|c|c|c|c|}
\hline DEG expression & TF count & $\mathrm{TF}$ & TAG count & TAG \\
\hline Downregulated & 3 & $S P I 1, P B X 3, L E F 1$ & 12 & $\begin{array}{l}\text { ONCO: WISP1, SPI1, HCK } \\
\text { TS: WNT5A, TP53INP1, } \\
\text { MMP11, GJB2, ENC1, CAPG } \\
\text { OTHER: PBX3, EVI2B, CTSZ }\end{array}$ \\
\hline Upregulated & 6 & $\begin{array}{l}\text { TCF7L2, PLAGL1, } \\
\text { NFIA, IRF1, EGR2, CEBPD }\end{array}$ & 17 & $\begin{array}{l}\text { ONCO: EGFR } \\
\text { TS: TGFBR3, TCF7L2, TCEAL7, SRPX, } \\
\text { SPRY2, SFRP1, PLAGL1, NDRG2, IRF1, } \\
\text { GPC3, EGR2, EEF1A1, CRABP1, CEBPD } \\
\text { OTHER: MSH6, CCL2 }\end{array}$ \\
\hline
\end{tabular}

ONCO, oncogene; TAG, tumor-associated gene; TF, transcription factor; TS, tumor suppressor.

Table IV. KEGG enriched pathway analysis for DEGs in the subnetwork.

\begin{tabular}{llc}
\hline KEGG pathway & $\mathrm{n}$ & P-value \\
\hline Staphylococcus aureus infection & 9 & $2.86 \times 10^{-11}$ \\
Osteoclast differentiation & 7 & $1.19 \times 10^{-05}$ \\
Natural killer cell-mediated cytotoxicity & 7 & $1.77 \times 10^{-05}$ \\
Cell adhesion molecules & 6 & $1.58 \times 1^{-04}$ \\
B cell receptor signaling pathway & 4 & $1.18 \times 10^{-03}$ \\
Antigen processing and presentation & 4 & $1.24 \times 10^{-03}$ \\
Systemic lupus erythematosus & 5 & $1.49 \times 10^{-03}$ \\
Rheumatoid arthritis & 4 & $2.41 \times 10^{-03}$ \\
Fc $\gamma$ receptor-mediated phagocytosis & 4 & $2.72 \times 10^{-03}$ \\
Chemokine signaling pathway & 5 & $6.19 \times 10^{-03}$ \\
Neuroactive ligand-receptor interaction & 6 & $6.48 \times 10^{-03}$ \\
Complement and coagulation cascades & 3 & $9.01 \times 10^{-03}$ \\
Viral myocarditis & 3 & $9.44 \times 10^{-03}$ \\
\hline
\end{tabular}

DEG, differentially expressed gene; KEGG, Kyoto Encyclopedia of Genes and Genomes.

PPI network of the DEGs. DEGs were integrated with interactions from the STRING database, and a PPI network was constructed (Fig. 1). The top 10 nodes with the highest degrees in the PPI network included: epidermal growth factor receptor (EGFR), degree=94; CD4, degree=43; lymphocyte cytosolic protein $2(\mathrm{LCP} 2)$, degree $=28$; hematopoietic cell kinase (HCK), degree=22; integrin $\beta 2$ (ITGB2), degree=21; interferon regulatory factor 1 (IRF1), degree $=21$; $\mathrm{C}-\mathrm{X}-\mathrm{C}$ motif chemokine $10($ CXCL10), degree=20; protachykinin 1 (TAC1), degree $=18$; TYRO protein tyrosine kinase-binding protein (TYROBP), degree=18; and C-C motif chemokine 2 (CCL2), degree $=16$. EGFR exhibited the highest degree, which suggested that it may serve a predominant role in breast cancer metastasis.

Subnetwork and KEGG functional analysis of DEGs. To further explore the key nodes for breast cancer metastasis, a subnetwork was established comprising 44 nodes using the BioNet tool, among which spleen tyrosine kinase (SYK) was predominant with the highest degree (degree=10; Fig. 2). KEGG pathway enrichment analysis indicated that proteins in the subnetwork, in which SYK was central, were involved in immune response pathways, such as Staphylococcus aureus infection, natural killer cell-mediated cytotoxicity and B cell receptor signaling pathway; as well as cell differentiation and cell adhesion pathways, including osteoclast differentiation and cell adhesion molecules (Table IV).

\section{Discussion}

The present study analyzed differential gene expressions, protein-protein interactions and GO term and KEGG pathway enrichment of the microarray profile GSE8977, and identified a total of 234 DEGs between normal and IDC breast tumor samples. The DEGs were revealed to be related to immune response, cell adhesion, proliferation and development-related functions and pathways. In addition, EGFR and SYK were identified as hub nodes in the PPI network or the subnetwork, respectively. Of the downregulated DEGs, three were identified as TFs, including SPII, lymphoid enhancer-binding factor 1 (LEF1) and pre-B-cell leukemia homeobox 3 (PBX3), of which $S P I 1$ is a known oncogene; two other downregulated genes, capping actin protein, gelsolin like $(C A P G)$ and tumor protein p53-inducible nuclear protein 1 (TP53INP1), were identified as TS genes. The upregulated DEGs also included TFs and TS genes, such as transcription factor 7-like 2 (TCF7L2) and pleiomorphic adenoma gene-like 1 (PLAGL1).

During the process of breast cancer metastasis, it is essential for the invasion of tumor cells to cause changes of cell adhesion, which allows them to invade into the surrounding host tissues (6). Thus, it is speculated that alteration of the genes and/or proteins that participate in cell adhesion or immune-related processes may have an influence on metastasis. A previous study has demonstrated that CAPG protein expression may inhibit tumorigenesis since they were lost at the stage of tumorigenesis in certain cancer cell lines, thus it has been proposed to be a TS gene (23). It has recently been reported as a potential biomarker for bone metastasis in breast 


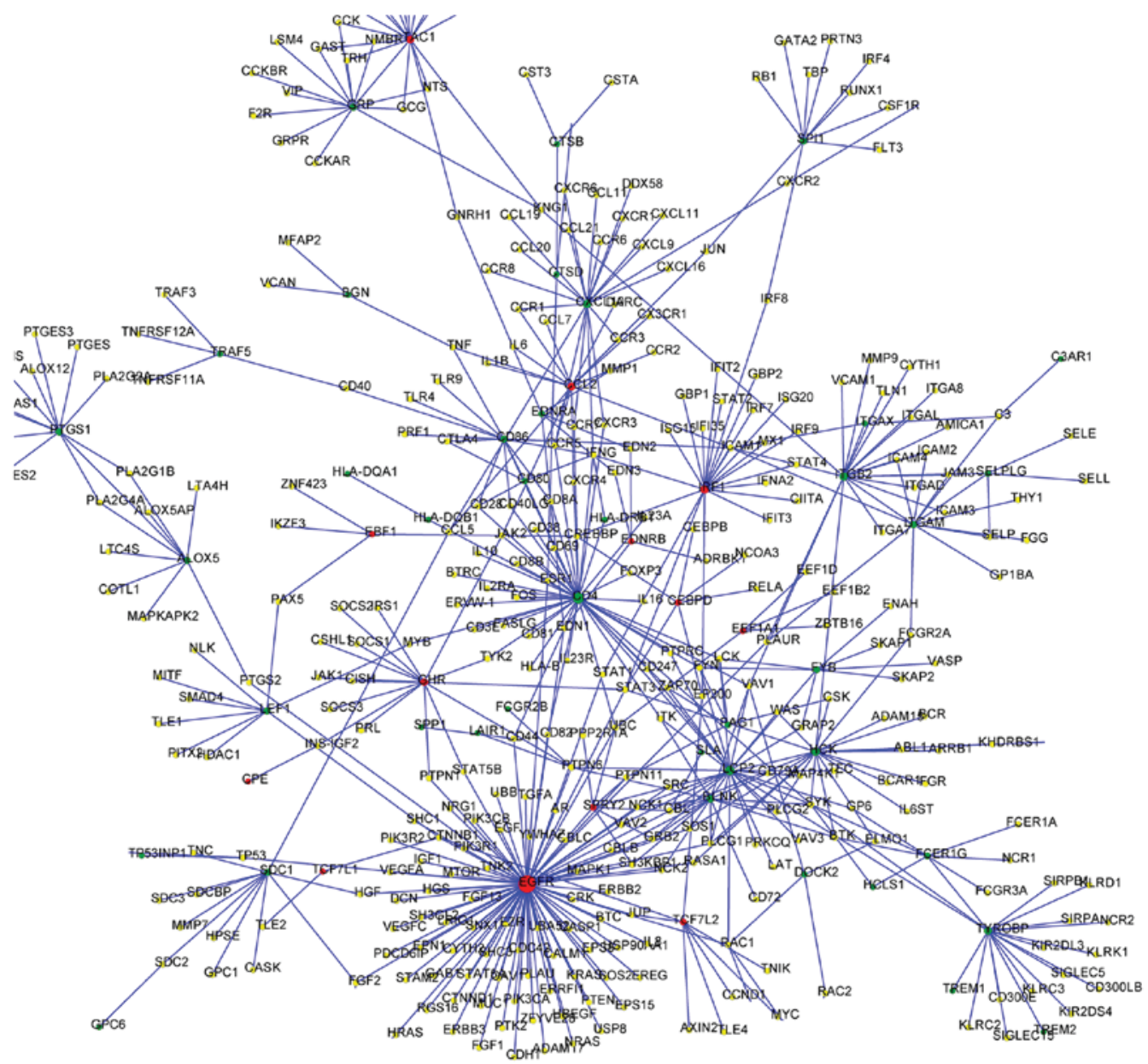

Figure 1. Predicted protein-protein interaction network of DEGs in breast cancer metastasis. Red nodes represent upregulated DEGs; green nodes represent downregulated DEGs; and yellow nodes represent genes that were not identified as differentially expressed. Blue lines represent the interaction relationships between two proteins. DEG, differentially expressed genes.

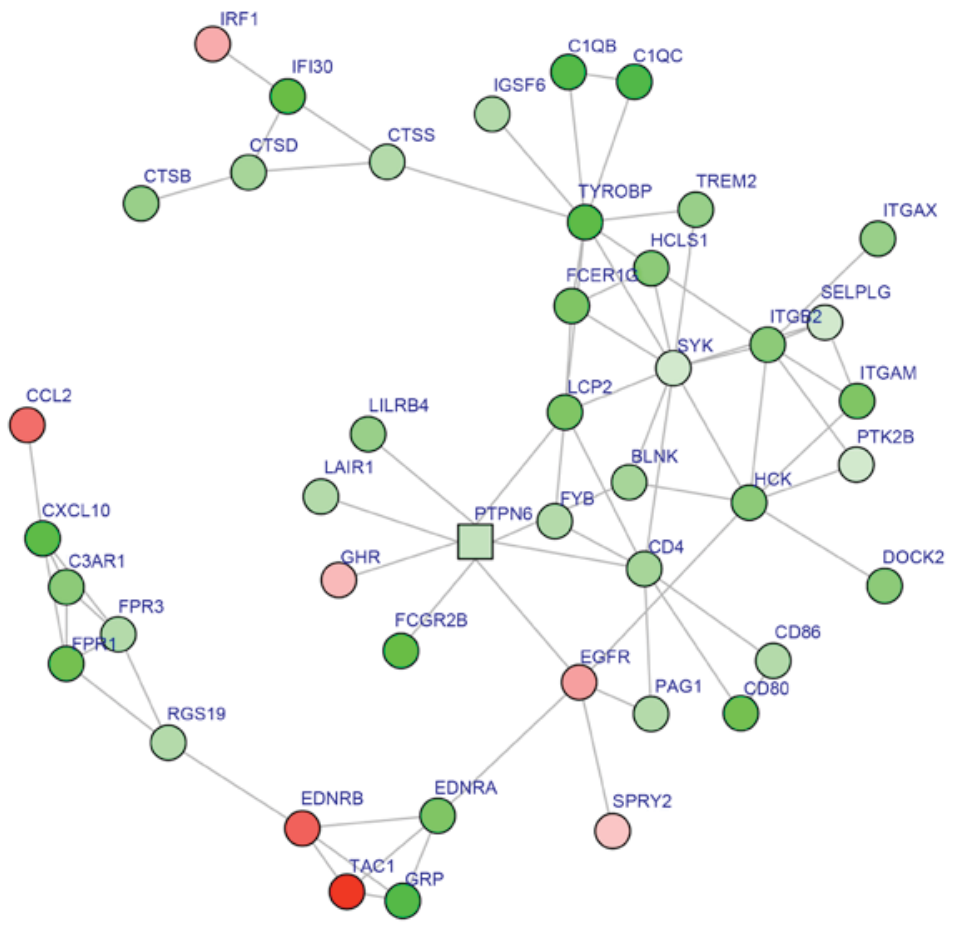

Figure 2. Subnetwork from the protein-protein interaction (PPI) network. Circles represent genes with high significance in the PPI network, and squares represent genes with low significance in the PPI network. Red nodes represent upregulated DEGs; green nodes represent downregulated DEGs; and pink nodes represent proteins with no significant expression change. Node color intensity was associated with the llog2 fold-changel of DEGs. DEG, differentially expressed gene. 
cancer (24). These results are confirmed by the present findings, which demonstrated that its expression was downregulated and was regarded as a TS gene in breast cancer metastasis. TP53INP1 is another downregulated TS gene identified by the present study, which has been reported to be associated with inflammation diseases (25). The expression level of TP53INPI was negatively associated with parameters such as tumor size, high histological grade, lymph node metastasis and aberrant p53 expression (26). TP53INP1 may suppress breast carcinoma since it was regulated by microRNA-34a which is a known suppressor of metastasis (27).

Previous in vivo experiments have verified that the downregulation of $P B X 3$ expression was able to suppress tumor growth and metastases (28). LEFI has been associated with the regulation of cell proliferation, and human breast cancer cells transfected with $L E F 1$ small interfering RNA resulted in a significant arrest at the G2/M phase of the cell cycle (29). SPII has been reported to be crucial for the differentiation or activation of macrophages or B cells (30). Macrophages and $B$ cells are involved in differentiation and immune-related processes, and they are found in the tumor microenvironment (30), thus may influence tumor progression. It may be inferred that the aforementioned TFs serve significant roles in breast cancer metastasis, and these roles may be through their involvement in the immune response. However, their potential targets need to be further explored.

Cell adhesion and proliferation are important processes that are associated with metastasis of various cancers $(31,32)$. PLAGL1 is a zinc-finger nuclear transcription factor that that has been reported to be involved in cellular proliferation in various cancer types (33). Enrichment analysis of the present study identified PLAGL1 as highly associated with the regulation of epithelial cell proliferation, which implied that it may participate in this function and, thus, may influence breast cancer metastasis. TCF $7 \mathrm{~L} 2$ has been implicated in colorectal cancer (34). The $\mathrm{T}$ cell factor $/ \beta$-catenin protein complex is oncogenic and serves significant roles in regulating cell adhesion, and TCF7L2 acts as an antagonist of this complex, which suggested the importance of TCF $7 L 2$ in cancer as a TS gene involved in cell adhesion. Notably, although the present study predicted TCF $7 L 2$ as a TS gene, its expression was revealed to be upregulated. On this basis, it was speculated that during breast cancer metastasis, TCF7L2 may serve a specific and different role compared with other, downregulated, TS genes. However, the inhibitory effects on metastasis by the upregulation of TCF7L2 expression may be small compared with the oncogenic roles of other genes. Nevertheless, it is likely that disturbed function of cell adhesion by TCF7L 2 might be important in the metastasis of breast cancer.

EGFR is a member of the type 1 family of cell surface receptor tyrosine kinases that has been correlated with numerous cancer types, including breast cancer (35). In addition, EGFR was demonstrated to be highly expressed in breast tumor cells with lymph node metastasis (36). Ephrin type-A receptor 2 (EPHA2) is a member of the Eph-receptor family and is commonly overexpressed in many types of cancers and contributes to the metastasis (37). EphA2 was reported to be induced by cell adhesion, and this increased expression was revealed to be dependent on the activation of EGFR (37). This result suggested the potential involvement of EGFR in cell adhesion. Combining these previous findings with the enrichment analysis of the present study, which demonstrated that $E G F R$ was an upregulated oncogene with the highest degree in the PPI network, it may be hypothesized that for breast cancer metastasis, EGFR may might exert its function by affecting cell adhesion, and high levels of $E G F R$ expression may be used as a strong prognosis gene.

SYK is a protein tyrosine kinase that is widely expressed in hematopoietic cells, and has been reported to have a close association with leukemia (38) and lymphoma (39). SYK expression was previously demonstrated to be common in normal human breast tissue; however its expression is low or undetectable in invasive breast carcinoma. In addition, SYK was revealed to function as a TS gene in breast cancer by reducing cell motility and invasion (40). Conversely, loss of SYK expression was reported to be associated with increased cellular invasiveness (41). Expression and signaling activity of $S Y K$ represents a favorable prognostic factor in breast cancer (42). SYK-regulated genes are important for breast cancer metastasis, as they may regulate immune responses, cell differentiation and cell adhesion pathways, which suggested that $S Y K$ may be used as an effective inhibitor for breast cancer metastasis.

In conclusion, the present study used a series of bioinformatics analyses to provide potential biomarkers such as $C A P G$, TP53INP1, SPI1, LEF1, PBX3, TCF7L2, PLAGL1, EGFR and $S Y K$ for breast cancer metastasis. Many of these DEGs such as LEF1, SPI1, PLAGL1 and TCF7L2 were associated with cell adhesion, cellular proliferation or the immune response and may influence the metastasis of breast cancer through the regulation of these function or pathways. However, these predictions require validation by in vitro and in vivo experiments. This study provides novel insights for breast cancer metastasis and suggests promising targets for inhibiting breast cancer metastasis.

\section{References}

1. Siegel R, Ma J, Zou Z and Jemal A: Cancer statistics, 2014. CA Cancer J Clin 64: 9-29, 2014.

2. Lockman PR, Mittapalli RK, Taskar KS, Rudraraju V, Gril B, Bohn KA, Adkins CE, Roberts A, Thorsheim HR, Gaasch JA, et al: Heterogeneous blood-tumor barrier permeability determines drug efficacy in experimental brain metastases of breast cancer. Clin Cancer Res 16: 5664-5678, 2010.

3. Kozlow W and Guise TA: Breast cancer metastasis to bone: Mechanisms of osteolysis and implications for therapy. J Mammary Gland Biol Neoplasia 10: 169-180, 2005.

4. Minn AJ, Gupta GP, Siegel PM, Bos PD, Shu W, Giri DD, Viale A, Olshen AB, Gerald WL and Massagué J: Genes that mediate breast cancer metastasis to lung. Nature 436: 518-524, 2005.

5. Weigelt B, Peterse JL and van't Veer LJ: Breast cancer metastasis: Markers and models. Nat Rev Cancer 5: 591-602, 2005.

6. Scully OJ, Bay BH, Yip G and Yu Y: Breast cancer metastasis. Cancer Genomics-Proteomics 9: 311-320, 2012.

7. Dontu G,El-Ashry D and Wicha MS: Breast cancer, stem/progenitor cells and the estrogen receptor. Trends Endocrinol Metab 15: 193-197, 2004.

8. Bhowmick NA, Neilson EG and Moses HL: Stromal fibroblasts in cancer initiation and progression. Nature 432: 332-337, 2004.

9. Slamon DJ, Leyland-Jones B, Shak S, Fuchs H, Paton V, Bajamonde A, Fleming T, Eiermann W, Wolter J, Pegram M, et al: Use of chemotherapy plus a monoclonal antibody against HER2 for metastatic breast cancer that overexpresses HER2. N Engl J Med 344: 783-792, 2001.

10. Hong B, Li H, Zhang M, Xu J, Lu Y, Zheng Y, Qian J, Chang JT, Yang $J$ and Yi Q: p38 MAPK inhibits breast cancer metastasis through regulation of stromal expansion. Int J Cancer 136: 34-43-2015. 
11. Phadke PA, Vaidya KS, Nash KT, Hurst DR and Welch DR: BRMS1 suppresses breast cancer experimental metastasis to multiple organs by inhibiting several steps of the metastatic process. Am J Pathol 172: 809-817, 2008

12. Karnoub AE, Dash AB, Vo AP, Sullivan A, Brooks MW, Bell GW, Richardson AL, Polyak K, Tubo R and Weinberg RA: Mesenchymal stem cells within tumour stroma promote breast cancer metastasis. Nature 449: 557-563, 2007.

13. Irizarry RA, Hobbs B, Collin F, Beazer-Barclay YD, Antonellis KJ, Scherf U and Speed TP: Exploration, normalization, and summaries of high density oligonucleotide array probe level data. Biostatistics 4: 249-264, 2003.

14. Gautier L, Cope L, Bolstad BM and Irizarry RA: affy-analysis of Affymetrix GeneChip data at the probe level. Bioinformatics 20: 307-315, 2004

15. Smyth GK: Limma: Linear models for microarray data. In Bioinformatics and computational biology solutions using $\mathrm{R}$ and Bioconductor Springer, pp397-420, 2005.

16. Ashburner M, Ball CA, Blake JA, Botstein D, Butler H, Cherry JM, Davis AP, Dolinski K, Dwight SS, Eppig JT, et al: Gene ontology: Tool for the unification of biology. The Gene Ontology Consortium. Nat Genet 25: 25-29, 2000.

17. Kanehisa M and Goto S: KEGG: Kyoto encyclopedia of genes and genomes. Nucleic Acids Res 28: 27-30, 2000.

18. Zhao M, Sun J and Zhao Z: TSGene: A web resource for tumor suppressor genes. Nucleic Acids Res 41: D970-D976, 2013.

19. Chen JS, Hung WS, Chan HH, Tsai SJ and Sun HS: In silico identification of oncogenic potential of fyn-related kinase in hepatocellular carcinoma. Bioinformatics 29: 420-427, 2013

20. Franceschini A, Szklarczyk D, Frankild S, Kuhn M, Simonovic M Roth A, Lin J, Minguez P, Bork P, von Mering C and Jensen LJ: STRING v9. 1: Protein-protein interaction networks, with increased coverage and integration. Nucleic Acids Res 41: D808-D815, 2013

21. Shannon P, Markiel A, Ozier O, Baliga NS, Wang JT, Ramage D, Amin N, Schwikowski B and Ideker T: Cytoscape: A software environment for integrated models of biomolecular interaction networks. Genome Res 13: 2498-2504, 2003.

22. Beisser D, Klau GW, Dandekar T, Müller T and Dittrich MT: BioNet: An R-Package for the functional analysis of biological networks. Bioinformatics 26: 1129-1130, 2010.

23. Watari A, Takaki K, Higashiyama S, Li Y, Satomi Y, Takao T, Tanemura A, Yamaguchi Y, Katayama I, Shimakage M, et al: Suppression of tumorigenicity, but not anchorage independence, of human cancer cells by new candidate tumor suppressor gene CapG. Oncogene 25: 7373-7380, 2006.

24. Westbrook JA, Cairns DA, Peng J, Speirs V, Hanby AM, Holen I, Wood SL, Ottewell PD, Marshall H, Banks RE, et al: CAPG and GIPC1: Breast cancer biomarkers for bone metastasis development and treatment. J Natl Cancer Inst 108, 2016.

25. Jiang PH, Motoo Y, Iovanna JL, Pébusque MJ, Xie MJ, Okada G and Sawabu N: Tumor protein $\mathrm{p} 53$-induced nuclear protein 1 (TP53INP1) in spontaneous chronic pancreatitis in the WBN/Kob rat: Drug effects on its expression in the pancreas. JOP 5: 205-216, 2004

26. Ito Y, Motoo Y, Yoshida H, Iovanna JL, Takamura Y, Miya A, Kuma K and Miyauchi A: Decreased expression of tumor protein p53-induced nuclear protein 1 (TP53INP1) in breast carcinoma. Anticancer Res 26: 4391-4395, 2006.

27. Peurala H, Greco D, Heikkinen T, Kaur S, Bartkova J, Jamshidi M, Aittomäki K, Heikkilä P, Bartek J, Blomqvist C, et al: MiR-34a expression has an effect for lower risk of metastasis and associates with expression patterns predicting clinical outcome in breast cancer. PLoS One 6: e26122, 2011.
28. Han HB, Gu J, Zuo HJ, Chen ZG, Zhao W, Li M, Ji DB, Lu YY and Zhang ZQ: Let-7c functions as a metastasis suppressor by targeting MMP11 and PBX3 in colorectal cancer. J Pathol 226: 544-555, 2012

29. Bucan V, Mandel K, Bertram C, Lazaridis A, Reimers K, Park-Simon TW, Vogt PM and Hass R: LEF-1 regulates proliferation and MMP-7 transcription in breast cancer cells. Genes Cells 17: 559-567, 2012

30. Lloberas J, Soler C and Celada A: The key role of PU.1/SPI-1 in B cells, myeloid cells and macrophages. Immunol Today 20 184-189, 1999.

31. Kannagi R, Izawa M, Koike T, Miyazaki $\mathrm{K}$ and Kimura N: Carbohydrate-mediated cell adhesion in cancer metastasis and angiogenesis. Cancer Sci 95: 377-384, 2004

32. Slack-Davis JK, Atkins KA, Harrer C, Hershey ED and Conaway M: Vascular cell adhesion molecule-1 is a regulator of ovarian cancer peritoneal metastasis. Cancer Res 69: 1469-1476, 2009.

33. $\mathrm{Yu} \mathrm{K}$, Lee $\mathrm{CH}$, Tan $\mathrm{PH}$ and Tan P: Conservation of breast cancer molecular subtypes and transcriptional patterns of tumor progression across distinct ethnic populations. Clin Cancer Res 10: 5508-5517, 2004.

34. Lepourcelet M, Chen YN, France DS, Wang H, Crews P, Petersen F, Bruseo C, Wood AW and Shivdasani RA: Small-molecule antagonists of the oncogenic Tcf/beta-catenin protein complex. Cancer Cell 5: 91-102, 2004.

35. Ferraro DA, Gaborit N, Maron R, Cohen-Dvashi H, Porat Z, Pareja F, Lavi S, Lindzen M, Ben-Chetrit N, Sela M and Yarden Y: Inhibition of triple-negative breast cancer models by combinations of antibodies to EGFR. Proc Natl Acad Sci USA 110: 1815-1820, 2013.

36. Liu Y, Ji R, Li J, Gu Q, Zhao X, Sun T, Wang J, Li J, Du Q and Sun B: Correlation effect of EGFR and CXCR4 and CCR7 chemokine receptors in predicting breast cancer metastasis and prognosis. J Exp Clin Cancer Res 29: 16, 2010.

37. Larsen AB, Stockhausen MT and Poulsen HS: Cell adhesion and EGFR activation regulate EphA2 expression in cancer. Cell Signal 22: 636-644, 2010

38. Goodman PA, Wood CM, Vassilev A, Mao C and Uckun FM: Spleen tyrosine kinase (Syk) deficiency in childhood pro-B cell acute lymphoblastic leukemia. Oncogene 20: 3969-3978, 2001.

39. Gururajan M, Dasu T, Shahidain S, Jennings CD, Robertson DA, Rangnekar VM and Bondada S: Spleen tyrosine kinase (Syk), a novel target of curcumin, is required for B lymphoma growth. J Immunol 178: 111-121, 2007.

40. Coopman PJ, Do MT, Barth M, Bowden ET, Hayes AJ, Basyuk E, Blancato JK, Vezza PR, McLeskey SW, Mangeat PH and Mueller SC: The Syk tyrosine kinase suppresses malignant growth of human breast cancer cells. Nature 406: 742-747, 2000.

41. Yuan Y, Liu H, Sahin A and Dai JL: Reactivation of SYK expression by inhibition of DNA methylation suppresses breast cancer cell invasiveness. Int J Cancer 113: 654-659, 2005.

42. Dejmek J, Leandersson K, Manjer J, Bjartell A, Emdin SO, Vogel WF, Landberg $G$ and Andersson T: Expression and signaling activity of Wnt-5a/discoidin domain receptor-1 and Syk plays distinct but decisive roles in breast cancer patient survival. Clin Cancer Res 11: 520-528, 2005. 